\title{
The Axial Vacuum Dynamic Sealing Technology for Rough Surface
}

\author{
Hao Guangping ${ }^{1,2}$, Liu Shaogang ${ }^{2}$, Zhou Guokui ${ }^{1}$ and Tang Dewei ${ }^{3}$ \\ 1.Harbin University of Science and Technology,Harbin, China \\ 2.Harbin Engineering University, Harbin, China \\ 3.Harbin Institute of Technology, Harbin, China
}

\begin{abstract}
A new axial vacuum dynamic sealing technology for rough surface, sliding dynamic sealing, is proposed, for the realization of well as the affecting factors. The conclusions that if the conditions are suitable, it is easy to realize the seal's rolling and sliding movement, and axial vacuum dynamic seal on cpartial vacuum chamber in hot-rolled pipe with continuous and efficient plasma descaling process. Based on simplified sealing model, the paper studies preliminarily this sealing mechanism, discusses the conditions of the seal implementation at the entrance and exit of the partial vacuum chamber, asertain rough surface can be achieved.
\end{abstract}

Keywords: rough surface, axial dynamic sealing, plasma descaling, partial vacuum chamber, rolling seal ring

\section{Introduction}

In the pipe production line, hot rolling process after the completion of the pipeline surface has a layer of hard, commonly known as the oxide scale. The existence of the scale, can accelerate the wear of the roll and the sizing machine roll. For this purpose, the pipe surface in the production of scale at the removal (descaling) is necessary. Ion cleaning technology since the mid- nineties [1] appears, many scholars have conducted indepth and extensive applied research [2,3]. Principle of plasma cleaning technology is primarily used plasma activation of high energy materials within will be attached to the removal of surface dirt thoroughly stripped $[4,5]$. Because of its environmentally friendly, integrated low cost, high quality cleaning, surface cleanliness can be achieved at the molecular level, regardless of the material, and covers an area of less advantages [ 5,6 ], it will become the preferred technology for descaling of metal.

The descaling process of high quality and efficiency should be done in less than $100 \mathrm{pa}$ vacuum $[8,9,7]$. The technology of wire descaling has begun to promote the use of abroad at present, descaling of pipe is in the experimental stage. Because of the difficulty of the rough surface axial vacuum dynamic sealing is larger, the technology in the domestic pipe descaling has no breakthrough. For this case, we propose a rough surface can be applied to the axial dynamic sealing technology, and the key technical problems were discussed.

\section{The Components of Plasma Cleaning Equipment}

The basic device of the plasma cleaning is shown in figure 1.It consists of the vacuum chamber, a vacuum pump, power supply, electrodes, a gas inlet part of the system, the workpiece transmission system and a control system, etc. [6 ] . 


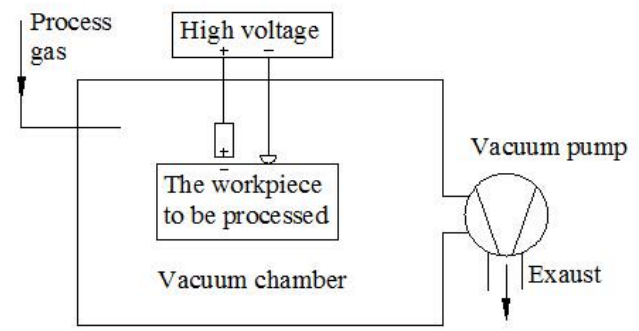

\section{Figure 1. Basic Device of Plasma Cleaning}

If a whole pipe was placed in a vacuum chamber, the plasma process is simple descaling,but when the longth of vacuum chamber of standard pipe is longer than $12 \mathrm{~m}$, causing its volume is too large, long-consuming vacuum extraction, and not conducive to the process in addition to continuous scales, and low efficiency. In order to solve the above drawbacks, as shown in Figure 2, we Can use a partial vacuum chamber to achieve protective gas atmosphere for plasma arc. That vacuum chamber axial freedom is constrained,then pipeline go through the vacuum chamber and axially move with uniform speed. the volume of the vacuum chamber is only required for plasma generating electrode. According to the working principle of the plasma descaling technology. is essential to keep descaling vacuum arc protective gas atmosphere to improve the efficiency and quality of descaling, therefore, reliable axial dynamic sealing of vacuum chamber and the rough surface ot the pipe is the key to achieving a vacuum atmosphere.

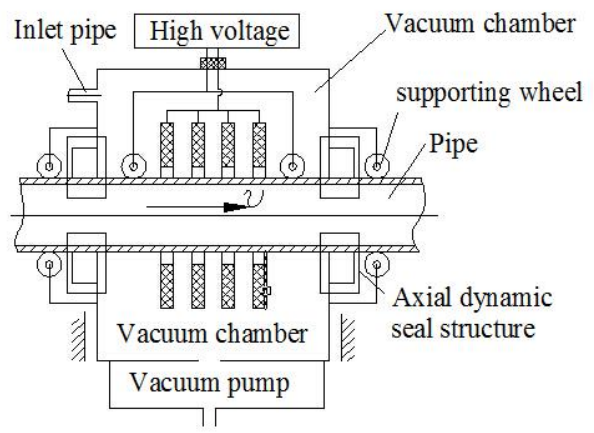

\section{Figure 2. Structure Diagram of Plasma Descaling for Pipe}

\section{Sealing Principle of Rolling and Sliding Type Dynamic Sealing}

To solve the axial dynamic sealing problems of rough surface of the pipeline, we proposed a rolling-sliding sealng method.the working state of the sealing ring cross section is shown in Figure 3. When the pipe move along the vacuum chamber of the axial, the seal ring produce pure rolling with the surface of pipe at point E. It also produce slippage with the surface of vacuum chamber. The vacuum chamber is easy to be sealed for that its outside surface is very smooth. The way of this sealing principle is that, the materials of seal ring are more soft than metal, so it has the characteristics of compliance. Parts of seal ring which are touching with the rough part of the pipe will deform along with unsmooth changing surface under the joint action of external pressure and mounting pressure, so it can achieve the purpose of sealing in this way. There are some irregular pits on the unprocessed rough surface. When the pits is light, the irregular pits can be filled by the amount of seal ring's deforming materials to achieve the purpose of seal. But when the pits is deep, there will be no enough materials filling the pit, so it cann't achieve the sealing purpose. This sealing method which achieve the sealing purpose by the pure rolling which is between the ring and the outer surface of rough pipe and the slippage 
which produce on the smooth outer surface of vacuum chamber. This sealing way also called 'Rolling and Sliding Seals'.

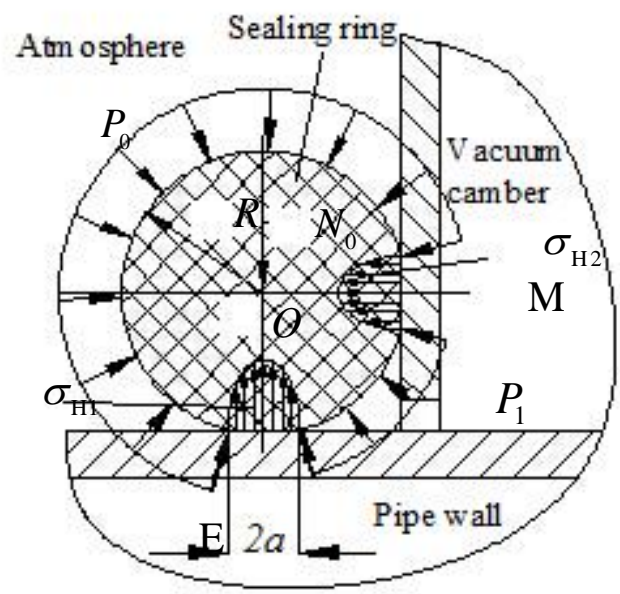

Figure 3. Cross Section Diagram of Seal Ring

$P_{0} \longrightarrow$ Atm

$P_{1} \_$Pressure of Vacuum Chamber

$R$ - Radius of Ring

$a-$ Under pressure, the half-width between the ring and torus of pipe

$\sigma_{\mathrm{H} 1}$ the contact stress between the under pressuring ring and the outside surface of pipe.

$\sigma_{\mathrm{H} 2}$ the contact stress between the under pressuring ring and vacuum wall

As shown in Figure 3, under the atmospheric and initial installation pressure, the pits can not be filled by seal ring's deforming materials, so the width of 2 a must bigger than the maximum axial dimension of pipe for achieving the sealing purpose.

\section{Conditions of the Rolling and Sliding Seals}

Using skid type axial dynamic seal, to achieve the realization of the sealing ring rolling sliding conditions were determined, in order to guide the selection of vacuum cavity sealing structure parameters. In the entrance and exit of the vacuum chamber, force of the sealing ring have bigger difference, therefore, it is necessary to discuss its separately skid implementation conditions. Based on the structural characteristics of seals, the seals by three research questions simplified into a two-dimensional. At any point on the stress, strain and deformation are only relevant to its location on the cross section, and has nothing to do with its axial position. Then we can simply study the ring cross section.

\subsection{The Entrance of the Rolling Sliding Conditions}

At the entrance, along the axial direction take one length for micro-analysis of field as an object, as shown in Figure 4. The micro section is mainly influenced by the static friction force of sliding friction, atmospheric pressure, vacuum press ure, pipe surface upward force of sealing ring, sealing cavity wall support of sealing ring, sealing ring flip torque caused itself. The torque is a function of the position of the flip ring, change periodically, it is defined as overturning moment . 


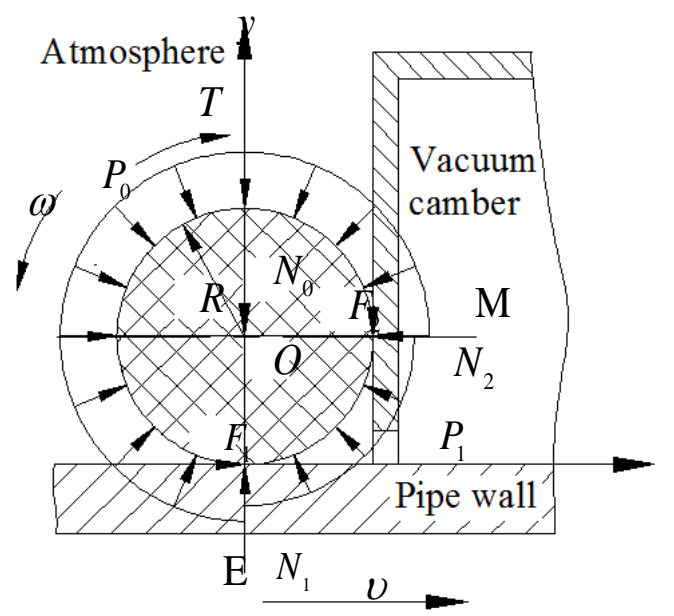

Figure 4. Force Analysis for Real Ring in Entrance

To facilitate the analysis, the atmospheric pressure and vacuum chamber pressure are reduced to $\Delta P$, its force situation as shown in Fig5 .

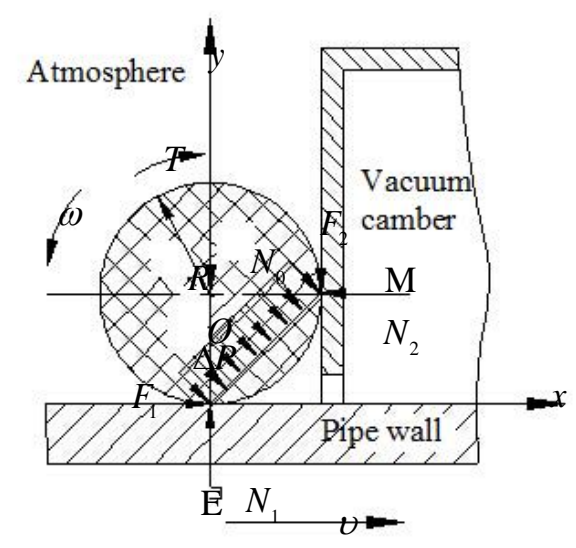

Figure 5. Force Model for Real Ring in Entrance

The action area of $\Delta P$ along the entire length of the seal ring is about

$$
S=\sqrt{2} \pi R(D+R)
$$

in the formula $D$ - Pipe diameter.

The pressure from $\Delta P$ on the vacuum chamber side walls and the outer surface of the pipe is approximately equal to

$$
F_{P}=\Delta P S \sin 45^{\circ}
$$

When pipe moves in uniform motion along the axial direction relative to the vacuum chamber, seals keep in balance, conclusion

$$
\left\{\begin{array}{l}
\left(F_{1}-F_{2}\right) R=T \\
F_{2}=\mu_{1} N_{2} \\
N_{2}=F_{P}+F_{1}
\end{array}\right.
$$

in the formula $\mu_{1} \_$sealing ring and the sliding friction coefficient of vacuum chamber wall .

namely 


$$
F_{1}=\frac{1}{1-\mu_{1}}\left(\mu_{1} F_{P}+\frac{T}{R}\right)
$$

This relationship explains the force that pipe wall in uniform motion to the rolling and sliding seals .

When overturning of the seals is maximum, static friction force pipes suffered is

$$
F_{1 \max }=\frac{1}{1-\mu_{1}}\left(\mu_{1} F_{P}+\frac{T_{\max }}{R}\right)
$$

In order to make rolling between seal and the surface of pipe, $F_{1 \max }$ must be less than the maximum static friction $F_{\text {max }}$ between seals and contact surfaces, namely

$$
F_{1 \max }<F_{\max }=\mu_{0} N_{1}=\mu_{0}\left(N_{0}+F_{P}\right)
$$

in the formula $\mu_{0}$ sealing ring and the static friction coefficient on the surface of the pipe ;

$N_{0}$ initial pressure of seal on the surface of the pipe after installation .

combine and simplify the formula $1 \sim 6$, conclusion

$$
\left(1-\mu_{1}\right) \mu_{0} N_{0}-\frac{T_{\max }}{R}>\left(\mu_{1}-\mu_{0}+\mu_{0} \mu_{1}\right) \pi R(D+R) \Delta P
$$

It's exactly the condition that when the seal ring rolls on the pipe surface, it also slides with vacuum vessel wall, namely roll sliding condition.

\subsection{The Exit Roll Slip Conditions}

At the outlet of the vacuum chamber, the pressure of seal ring is shown in Figure 6 .

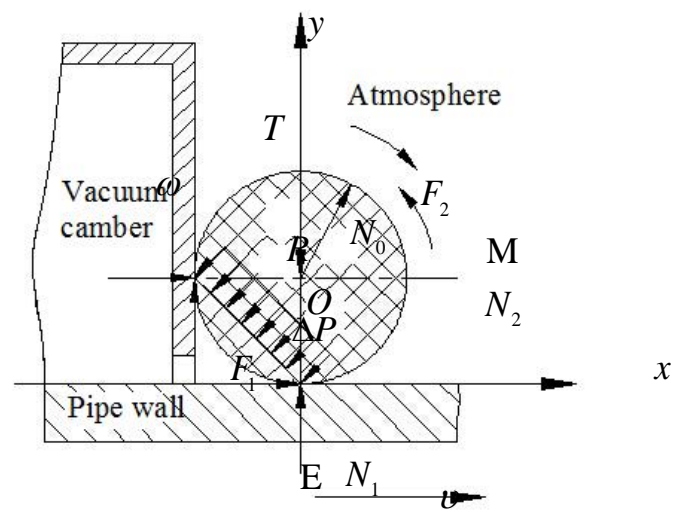

Figure 6. Force Simplified Model for Real Ring in Exit

When pipeline is in uniform motion, as the balance of seal ring, conclusion

namely

$$
\left\{\begin{array}{l}
\left(F_{1}-F_{2}\right) R=T \\
F_{2}=\mu_{1} N_{2} \\
N_{2}=F_{P}-F_{1}
\end{array}\right.
$$

$$
F_{1}=\frac{1}{1+\mu_{1}}\left(\mu_{1} F_{p}+\frac{T}{R}\right)
$$


The same can be concluded that the condition for seals rolling and sliding at the outlet of vacuum chamber is

$$
\left(1+\mu_{1}\right) \mu_{0} N_{0}-\frac{T_{\max }}{R}>\left(\mu_{1}-\mu_{0}-\mu_{0} \mu_{1}\right) \pi R(D+R) \Delta P
$$

\subsection{The Error Derived from the Simplified Impact Roller Sliding Conditions}

The size of the deformation of the seal ring has a direct effect on its leakproofness . Calculate the width of the contact surface after deformation and maximum contact stress according to Hertz theory .

When the seal ring in the atmospheric pressure $\mathrm{P} 0$ and the effect of internal pressure $\mathrm{P} 1$ , contact with the outer surface of the pipe and the surface of the vacuum vessel wall is actually surface contact . Because of the large diameter of the pipe, the contact of seals and outer surfaces of the pipe can be simplified to the contact of cylinders and flat contacts. The size of the contact area and contact stress can approximate according to Hertz contact theory. Under the force $\Delta P$, the situation of pipe outer surface stress is similar to the force of the cavity wall, simplified model is shown in figure 7 .

Based on Hertz contact theory, the Half-width of the contact surface a is

$$
a=\sqrt{\frac{4 F_{P}}{\pi b} \rho\left(\frac{1-v^{2}}{E}\right)}
$$

in the formula $E$ — elasticity modulus of seal material ;

$v-$ Poisson's ratio of seal ring material ;

$b$ - The length of the contact surface, $b=\pi D$;

$\rho-$ The radius of curvature seal ring, $\rho=R$.

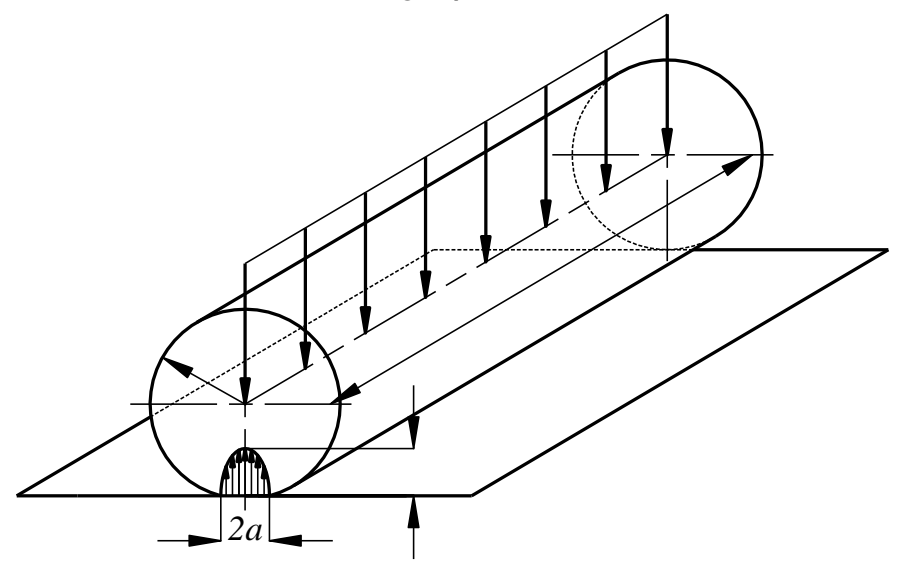

Figure 7. Simplified Model for Contact of Real Ring

Plug formula 2 in formula 11 and simplify, the Half-width of the contact surface produced by the effect of $\Delta P$ is

$$
a=2 R \sqrt{\frac{\Delta P(D+R)}{\pi D}\left(\frac{1-v^{2}}{E}\right)}
$$

Take the rubber elastic modulus $E=7.84 \mathrm{MPa} 、$, Poisson's ratio $v=0.47 、 \Delta P$ is a pressure , pipe diameter $D=1200 \mathrm{~mm}$, radius of the seal ring cross-section are $10 \mathrm{~mm}$ 、 $20 \mathrm{~mm} 、 30 \mathrm{~mm}$ respectively, results are shown in Table 1 calculated by formula 12 . 
Table 1. Calculation Results of Half Width for Contact Surface

\begin{tabular}{c|c}
\hline Seal sectional radius $R / \mathrm{mm}$ & Half-width of the contact surface $a / \mathrm{mm}$ \\
\hline 10 & 1.14 \\
20 & 2.28 \\
30 & 3.43 \\
\hline
\end{tabular}

Radial deformation of the seal ring is defined as $\xi$

$$
\xi=\frac{R-\sqrt{R^{2}-a^{2}}}{R} \times 100 \%
$$

Plug the data of table 1 in formula 13 to calculate respectively, the radial deformation $\xi$ of the seal ring volume above is about $0.65 \%$, during the theoretical derivation of rolling and sliding conditions the impact of the modification can be ignored.

Considering of Table 1 and the atmospheric pressure, when the seal radius is $20 \mathrm{~mm}$ and pipe outside diameter is $1200 \mathrm{~mm}$ and the ectotheca of vacuum chamber is exactly right perpendicular to the direction of pipeline motion, the width is about $4.56 \mathrm{~mm}$ between sealing and the contact ring of outside surface.It means that any pits whose axial dimension are less than $4.56 \mathrm{~mm}$ can be covered to achieve the purpose of sealing.

\subsection{The Influence Factor of Rolling Condition}

Analyzed formula 8 and formula 9,it comes the fact that the condition of skiding is connected with the following parameters:the pressure to pipe surface after stretching the seal ring which is $N_{0}$, the maximum rolling torque which is $T_{\max }$, the coefficient of dynamic friction between the side wall of vacuum chamber and seal ring which is $\mu_{1}$, the sectionalradius of seal ring which is $R$, the coefficient of static friction between pipe and seal ring which is $\mu_{0}$ 。 It is easy to meet the condition of rolling, as long as adjust the parameter reasonably。 Therefore, rolling seal is feasible in theory。

\section{Conclusion}

Under the background of plasma continuously wiping scale of pipe the author present a vacuum seal technique which uses pure rolling to solve the problem of rough surface axial direction vacuum motive seal.This article mainly analyzes the mechanism of rolling motive seal,the rolling conditions for seal ring and its influence factor in theory.; the analysis shows that it is easy to meet the condition of rolling,as long as adjust the parameter reasonably,Presenting this technique will solve the key to the question of plasma continuously wiping scale of pipe,thus it advances this technique in China.

\section{Acknowledgments}

This paper is Supported by China Postdoctoral Science Foundation (20090460879).

\section{References}

[1] D. Q. Zhou, "New Technology of Vacuum Arc Discharge", CAAI Transactions on Intelligent Systems, vol. 2, no. 9, (2001).

[2] N. Suzuki, T. Sasaoka and K. Tomita, "Plasma cleaning machine and its applications", Matsushita Technical Journal, vol. 4, (2003), pp. 53-61

[3] B. Kegel and H. Schmid, "Low-pressure plasma cleaning of metallic surfaces on industrial scale", Surface and Coatings Technology, vol. 1, no. 112, (1999), pp. 63-66

[4] PLASMAtech.inc Plasma Surface Modification of Ploymers.

[5] T. Zhang, Technical Research of Using Plasma”, Equipment for Electronic Products Manufacturing, vol. 7, no. 137, (2006), pp. 21-27 
International Journal of Control and Automation

Vol. 8, No.4 (2015)

[6] C. C. Xiong, "Plasma cleaning technique", cleaning technique, no. 1, pp. 41-44

[7] W. Petasch, B. Kegel and H. Schmid, "Low-pressure plasma cleaning: a process for precision cleaning applications", Surface and Coatings Technology, vol. 12, no. 97, (1997), pp. 176-181.

[8] P. Fuchs, "Low-pressure plasma cleaning of Au and PtIr noble metal surfaces", Applied Surface Science, vol. 256, (2009), pp. 1382-1390.

[9] W. W. Yang, H. W. Du, D. W. Wang and Y. X. Xu, "Research of Low Pressure Plasma Cleaning Inline", Electronics Process Technology, vol. 1, no. 32, (2011), pp. 12-15 